\title{
MANEJO FISIOTERAPÊUTICO AO PACIENTE COM COVID-19 EM UNIDADE DE TERAPIA INTENSIVA
}

\author{
ARTIGO ORIGINAL \\ PEREIRA, Holda Maria de Jesus ${ }^{1}$ \\ OLIVEIRA, Tatiane Alves Cortez de ${ }^{2}$ \\ ALMEIDA, Leandro Augusto ${ }^{3}$
}

PEREIRA, Holda Maria de Jesus. OLIVEIRA, Tatiane Alves Cortez de. ALMEIDA, Leandro Augusto. Manejo fisioterapêutico ao paciente com COVID-19 em Unidade de Terapia Intensiva. Revista Científica Multidisciplinar Núcleo do Conhecimento. Ano 06, Ed. 01, Vol. 01, pp. 123-138. Janeiro de 2021. ISSN: 2448-0959, Link de acesso: https://www.nucleodoconhecimento.com.br/saude/manejo-fisioterapeutico

\section{RESUMO}

O Coronavírus (SARS-COV-2) contaminou muita gente no Brasil e no mundo. A doença mostrou importância do profissional de fisioterapia estar atualizado e ter conhecimento das técnicas e recursos para o manejo correto dos pacientes dentro da Unidade de Terapia Intensiva. Este estudo teve como objetivo geral delinear a assistência fisioterapêutica ao paciente com COVID-19 na unidade de terapia intensiva. Trata-se de uma revisão bibliográfica, baseada em análise de artigos científicos publicados no ano de 2020. A busca de dados foi realizada no período de março a setembro de 2020, onde foram selecionados artigos em conformidade com o

1 Fisioterapeuta. Instituto Educacional Santa Catarina - Faculdade Guaraí (IESC/FAG). Curso de Fisioterapia.

2 Fisioterapeuta. Instituto Educacional Santa Catarina Faculdade Guaraí (IESC/FAG). Curso de Fisioterapia.

${ }^{3}$ Mestre em Bioengenharia com Ênfase na Saúde. Fisioterapeuta. 
tema proposto, sendo realizado um levantamento bibliográfico nas bases de dados: Scientific Electronic Library Online (SciELO), Literatura Latina Americana em Ciência de Saúde (LILACS), Google Acadêmico, Physiotherapy Evidence Database (PEDro) e PubMed. Apesar de grande parte da população infectada apresentarem apenas sintomas leves, alguns podem ter uma forma mais grave da doença sendo necessário a hospitalização em um leito clínico ou até mesmo em Unidade de Terapia Intensiva, pois alguns casos de pacientes com COVID-19 pode evoluir para diferentes níveis de gravidade da doença, podendo levar a uma insuficiência respiratória aguda (IRA), a síndrome do desconforto respiratório aguda (SDRA) ou até a falência de múltiplos órgãos. Sendo assim, conclui-se que o atendimento fisioterapêutico é de extrema importância para o paciente, tanto na admissão como durante todo o tempo de internação na unidade de terapia intensiva.

Palavras-chave: COVID-19, Síndrome do Desconforto Respiratório Agudo, Unidade de Terapia Intensiva, Fisioterapia, Coronavírus.

\section{INTRODUÇÃO}

Em dezembro de 2019, uma nova epidemia surgiu na cidade de Wuhan e se propagou por toda a China. Tratava-se inicialmente de uma pneumonia de origem etiológica desconhecida e que por meio de um sequenciamento genético, foi classificado como um novo gênero beta coronavírus, sendo o mesmo definido como nova pneumonia pela Organização Mundial de Saúde (OMS) (SILVA et al., 2020).

Os sintomas são parecidos aos de uma gripe comum, no entanto, pacientes com essa patologia podem precisar de cuidados intensivos (MATOS e SCHAPER, 2020). De acordo com um estudo feito pelo Center for Diseases Control and Prevention dos Estados Unidos, com base em dados da China, o índice de mortalidade é maior em idosos, em especial aqueles que apresentavam alguma doença subjacente (IMOTO et al., 2020).

Apesar da maioria dos pacientes apresentarem sintomas leves como febre, tosse seca e dor de garganta, alguns evoluíram para maiores complicações, como falência de 
órgãos, edema pulmonar, choque séptico, quadro de pneumonia grave e Síndrome do Desconforto Respiratório Agudo (SDRA) (SOHRABI et al., 2020). Segundo (SILVA et al., 2020) os comprometimentos desse vírus vão além do sistema respiratório, agravando também outros sistemas, como o cardiovascular, causando uma descompensação desse sistema, principalmente em pacientes com doença arterial coronariana e insuficiência cardíaca.

A transmissão do vírus acontece principalmente através das vias respiratórias, mediante a inalação de gotículas, eliminadas por meio do espirro ou tosse, durante procedimentos como a ventilação não invasiva (VNI), intubação e extubação, aspiração, ressuscitação cardiopulmonar, e broncoscopia (FRESCA et al., 2020).

Diante do índice elevado de pessoas contaminadas, da rápida propagação do vírus e do número expressivo de pessoas que precisam de cuidados em uma UTI, a problemática estabelecida nesta revisão é: como a fisioterapia pode prestar uma assistência de qualidades a esses pacientes? Pois dentre os vários profissionais que atuam para promover a recuperação do paciente com COVID-19, destaca-se o fisioterapeuta. Suas atribuições visam prevenir e reabilitar os problemas respiratórios e as limitações funcionais das atividades de vida diárias (AVDs) por ela causada (SILVA et al., 2020).

A fisioterapia respiratória em enfermarias e Unidade de Terapia Intensiva (UTI) pode ser indicada para pacientes com suspeita ou em caso confirmado que conjuntamente ou posteriormente desenvolvam hipersecreção, consolidações pulmonares exsudativas e/ou dificuldade para remoção de secreções de vias aéreas (MATOS, 2020).

A necessidade de intubação e ventilação mecânica invasiva (VMI) geralmente são necessárias, pois alguns pacientes falham na oxigenoterapia e na Ventilação não Invasiva (VNI) ou já tem indicação direta de VM e precisam rapidamente serem intubados (SILVA et al., 2020). 
Trata-se de uma revisão de bibliográfica, baseada em análise de artigos científicos publicados no ano de 2020. A coleta de dados foi realizada no período de março a julho de 2020, selecionando artigos em conformidade com o assunto proposto, através de levantamento bibliográfico nas bases de dados eletrônicas Scientific Electronic Library Online (SciELO), Literatura Latina Americana em Ciência de Saúde (LILACS), Google Acadêmico, Physiotherapy Evidence Database (PEDro) e PubMed. Os Descritores em Ciências da Saúde (DCS) utilizados serão COVID-19, síndrome do desconforto respiratório agudo, unidade de terapia intensiva, fisioterapia e ventilação mecânica e seus correlatos na língua inglesa acute respiratory distress syndrome, intensive care unit, physiotherapy and mechanical ventilation. Os critérios de inclusão determinados serão artigos nas línguas portuguesa e inglesa relacionados ao tema e publicados este ano.

\section{DESENVOLVIMENTO}

\section{PERFIL HISTÓRICO DA COVID-19}

A atual pandemia causada pelo coronavírus 2019 (SARS CoV-2), tem provocado sérios danos para os Sistemas de Saúde no Brasil e no mundo (PINTO e CARVALHO, 2020). A Organização Mundial de Saúde (OMS) declarou em 11 de março de 2020, que o surto se tratava de uma pandemia. O primeiro caso confirmado no Brasil foi em 25 de fevereiro de 2020 com aumento contínuo de pessoas acometidas em vários estados brasileiros (GUIMARÃES, 2020).

A rápida propagação do vírus de forma mundial e o crescente números de casos, levou os Sistemas de Saúde ao colapso, devido ao número insuficiente de profissionais de saúde, falta de leitos em unidades de terapia intensiva (UTI) para atender ao aumento da demanda e a falta de ventiladores mecânicos (WITTMER et al., 2020).

Ao mesmo tempo que o mundo luta para controlar o avanço da doença, uma grande tensão para comportar o crescente número de doentes têm afetado às cidades. Isso 
tem feito com que medidas extremas sejam tomadas para amenizar a crise de leitos e a falta de profissionais em hospitais (YEO et al., 2020).

Tal pandemia mostrou como um vírus bastante infeccioso é capaz de sobrecarregar os Sistemas de Saúde até mesmo de países economicamente desenvolvidos, sendo um dos principais agravantes para que isso ocorra a falta de leitos nas UTIs e a falta de recursos para o tratamento de pacientes em ventilação mecânica (SEVERIN et al., 2020).

\section{PAPEL DO FISIOTERAPEUTA NA COVID-19}

Diante da crise que se instalou no mundo decorrente do avanço do novo coronavírus, evidenciou a importância da equipe multidisciplinar de saúde, entre os quais podemos enfatizar a importância da atuação do fisioterapeuta na linha de frente da assistência aos pacientes suspeitos de infecção ou mesmo contaminados pelo SARSCoV-2 (COSTA e LOIOLA, 2020).

Por se tratar de uma doença nova onde não houve tempo suficiente para estudos clínicos e revisões sistemáticas que pudesse direcionar as intervenções, o tratamento tem sido efetuado a partir da vivência de países que já enfrentaram ou ainda estão enfrentando um número de casos elevados, bem como em estudos sobre a Síndrome do Desconforto Respiratório Agudo (SDRA), a Síndrome Respiratória Aguda Grave (causada pelo coronavírus SARS-CoV) e na Síndrome Respiratória do Oriente Médio (causada pelo coronavírus MERS- CoV) (GUIMARÃES, 2020).

Conhecer os sinais e sintomas é uma condição decisiva para a escolha das técnicas fisioterapêuticas e dos recursos a serem utilizados, no entanto, o atendimento deverá ser planejado e adequado de acordo com as condições ecossistêmicas disponíveis, considerando que há um grande risco de dissipação do vírus (FURLANETTO et al., 2020).

Estudos já publicados sobre a atuação do fisioterapeuta no contexto dessa patologia abordam a importância desse profissional estar atualizado e possuir a competência 
necessária para prestar uma assistência de qualidade nos variados níveis de gravidade do paciente, no manejo da ventilação mecânica invasiva e não invasiva, durante a oxigenoterapia, nas técnicas para a atenuação da geração de aerossóis, nos cuidados com pacientes hipersecretivos, a pacientes com comprometimento do sistema respiratório ou com dispneia, entre outras comorbidades.

Apesar de algumas publicações direcionarem o atendimento aos pacientes com estado de saúde mais grave, não indicando o atendimento fisioterapêutico caso o paciente com COVID-19 apresente sintomas leves em que não há um comprometimento expressivo do aparelho respiratório, atualmente o atendimento fisioterapêutico se tornou imprescindível durante o processo de internação, atuando na prevenção e no tratamento dos distúrbios respiratórios consequentes da retenção de secreção e de atelectasias, assim como na preservação da força muscular e funcionalidade no tempo de hospitalização, auxiliando na intubação, nos ajustes do ventilador mecânico - programando os parâmetros ventilatórios iniciais - na monitorização da mecânica respiratória, no desmame da ventilação mecânica (VM) e durante a extubação.

Assim sendo, é recomendado a atuação de profissionais peritos e/ou com experiência, titulação e/ou treinamento dedicado em Terapia Intensiva e/ou Fisioterapia Respiratória, além de conhecimento dos recursos disponíveis (MUSUMECI et al., 2020). Pois assim como as alterações pulmonares requerem uma atenção significativa, os efeitos do tempo decorrido de imobilidade no leito do mesmo modo. Portanto os exercícios devem ser incluídos o mais precocemente possível, sejam eles exercícios passivos, ativos-assistidos, ativos, eletroestimulação, posicionamento no leito, exercícios respiratórios, com a intenção de reduzir ao máximo a perda da capacidade funcional do paciente e uma melhor recuperação da qualidade de vida após a infecção (MATTE et al, 2020).

Espera -se que alguns resultados possam ser alcançados, entre eles:

- Redução do número de intubações; 
- Redução no tempo de internação na UTI e na VM;

- Redução do número de infeções respiratórias;

- Redução no índice de mortalidade ao longo do período de internação após a alta hospitalar.

No decorrer do tratamento há um possível risco de transmissão aérea do vírus causador do COVID-19, pois algumas técnicas empregadas com objetivo de melhorar as disfunções respiratórias são grandes geradores de aerossóis, os quais podemos destacar a hiperinsuflação manual, treinamento muscular respiratório e VNI. Portanto, é preciso avaliar cuidadosamente a recomendação e o emprego desses recursos, onde ocorre um maior risco de contaminação (MUSUMECI et al., 2020).

Em relação às técnicas usadas pelo fisioterapeuta uma outra preocupação está voltada para o aumento do gasto energético excessivo, principalmente em pacientes em estado grave. Pois essa situação causado por mobilizações ativas ou até mesmo pelo exercício físico pode agravar os músculos respiratórios e gerar um quadro de parada respiratória (FURLANETTO, 2020).

\section{VENTILAÇÃO NÃO INVASIVA}

De acordo com algumas publicações o uso da VNI não é recomendado como uma estratégia ventilatória de primeira linha, devido ao alto percentual de falha e ao aumento da disseminação viral consequente da geração de aerossóis no ambiente ocasionado por algumas técnicas como a hiperinsuflação com ressuscitador manual e o uso da cânula nasal de alto fluxo (CNAF). Porém, em alguns casos, definidos pela equipe multidisciplinar, um teste de resposta a VNI pode ser realizado, durante 30 minutos, desde que o paciente apresente uma saturação periférica de oxigênio $\left(\mathrm{SPO}_{2}\right)$ $<93 \%$ no cateter nasal a $51 /$ min (MARTINEZ et al., 2020).

O teste pode ser realizado desde que haja circunstâncias ideais para sua execução, como a utilização de máscara sem válvula de exalação acoplada a circuitos ventilatórios com ramo duplo, assim como a utilização correta dos filtros trocadores de calor e umidade, forma semelhante ao padrão utilizado na VM dos filtros e dos 
dispositivos de umidificação passiva e equipamentos de proteção individual (EPIs) (MARTINEZ et al., 2020).

Em casos em que o uso da VNI é indicado, o paciente deve ser monitorado cuidadosamente, e se não houver uma melhora dos padrões clínicos é necessário alertar a equipe quanto a indicação da VMI, pois o retardo na intubação pelo uso demorado da VNI pode estar relacionado a grande taxa de mortalidade, sobretudo em pacientes mais graves (MUSUMECl et al., 2020).

Como visto anteriormente, existem autores em suas publicações que não recomendam o uso da VNI ou da cânula nasal de alto fluxo (HFNC). Porém, algumas experiências realizadas na China recomendam o uso da VNI e da HFNC de forma precoce, podem gerar uma taxa de mortalidade mais reduzida e a diminuição nos casos de intubação (WINCK e AMBROSINO, 2020).

Apesar das incertezas e da falta de evidências científicas, os resultados positivos em grande parte dos estudos observacionais já sugerem que em um ambiente seguro, como uma sala de pressão negativa, com pacientes criteriosamente avaliados e sem nenhuma indicação de VMI, a utilização da VNI pode ser considerada (GÓMEZ et al., 2020).

Dessa forma, o uso da VNI como tratamento deve ser um recurso a ser utilizado desde que haja um acompanhamento criterioso para constatar se há risco de falha ou se é necessário realizar a mudança para uma VMI.

\section{VENTILAÇÃO MECÂNICA}

É importante observar sinais de insuficiência respiratória hipoxêmica, pois o aumento do trabalho respiratório e o baixo nível de oxigênio no sangue pode piorar gradualmente, apesar do uso da oxigenoterapia convencional e a intubação endotraqueal deve ser realizada o mais rápido possível (BLANCO e DUDARYK, 2020). A equipe de saúde deve fazer uso de EPls (equipamento de proteção individual) como luvas, capote, touca, máscara protetora (N95/ PFF2), além de óculos 
ou protetor facial. Além do mais, os pacientes com insuficiência respiratória grave que necessitam de suporte ventilatório elevado, pois podem progredir rapidamente de uma ventilação espontânea para a necessidade de intubação e ventilação mecânica, o que pode durar por até 3 semanas. (GUIMARÃES, 2020).

Durante a VMI deve-se optar por uma estratégia de ventilação protetora, com objetivo de diminuir o risco de lesão pulmonar ocasionado pela ventilação, por isso é recomendado:

- Modo ventilatório controlado a volume (VCV) ou a pressão (PCV);

- Manutenção da pressão de distensão (driving pressure) inferior a $15 \mathrm{cmH}_{2} \mathrm{O}$;

- De forma individualizada realizar o ajuste da pressão positiva expiratória final (PEEP);

- Tolerar uma hipercapnia permissiva;

- Uma frequência respiratória próxima do ideal de 20 irpm.

- Programar uma FiO2 de 92 a 96\% (REIS, 2020).

Estudos já demonstraram que o uso de parâmetros elevados de volume e pressão podem ser fatores causadores de injúria pulmonar e estão relacionados a um número maior de mortos. Sendo assim, pacientes que apresentarem um quadro clínico de SDRA devem ser priorizados uma estratégia ventilatória protetora (FURLANETTO, 2020).

\section{DESMAME E EXTUBAÇÃO}

O processo de extubação do paciente envolve a transição gradual da ventilação artificial para a ventilação espontânea, feita com pacientes em ventilação mecânica invasiva por um tempo superior a 24 horas. Diante disso, é realizado um teste de ventilação espontânea com auxílio de um tubo endotraqueal que permite que o paciente ventile por si só. Os pacientes com resultados satisfatórios no teste devem ser analisados quanto a indicação de retirada do paciente do ventilador mecânico (BRANCO E ARRUDA, 2020). A Organização Mundial de Saúde recomenda a utilização de protocolos de desmame onde possibilita a respiração espontânea seja 
diariamente avaliada, sempre que o quadro clínico do paciente permitir. Para que o processo de desmame ocorra, deve ser considerado as seguintes condições (CASTRO et al., 2020):

- Nível de consciência (escala de Coma de Glasgow $\geq 8$ ).

- Oxigenação adequada: $\mathrm{PaO}_{2} \geq 60 \mathrm{mmHg}$ com $\mathrm{FiO}_{2} \leq 0,40$ e Peep $<10$.

- Ausência de hipersecreção (mais do que 1 aspiração a cada 2 horas).

- Estabilidade hemodinâmica: pressão arterial média $\geq 60 \mathrm{mmHg}$ sem necessidade de vasopressores (ou em doses baixas).

- Capacidade de proteção de vias aéreas: pico de fluxo expiratório > $60 \mathrm{~L} / \mathrm{min}$.

- Não apresentar suspeita de edema de vias aéreas.

Na hipótese de falha no desmame, é importante investigar o que levou a falha. Avaliar o quadro geral do paciente, a estabilidade hemodinâmica, sinais de esforço ventilatório, se necessário realizar novas avaliações. Antes de uma nova tentativa de desmame recomenda-se aguardar 24 horas (CASTRO et al., 2020).

\section{POSIÇÃO PRONA}

Como alguns pacientes têm a função pulmonar prejudicada rapidamente causando uma grave hipoxemia e sérias alterações nas trocas gasosas, é necessário um tratamento imediato. Dessa forma muitas unidades de atendimento começaram a tratar os pacientes na posição prona, e em alguns casos, os níveis de oxigenação aumentaram significativamente. Apesar de nem todos apresentarem uma melhora, há relatos de pacientes com COVID-19 que foram tratados em prono e obtiveram bons resultados no tratamento. Por isso, muitos centros passaram a oferecer precocemente a pronação como uma alternativa para pacientes com respiração espontânea, antes mesmo da intubação e da ventilação mecânica (LINDAHL, 2020).

A posição prona é um dos recursos terapêuticos que se destaca no tratamento do doente com SDRA. O método consiste em deitar o paciente em decúbito ventral, com objetivo de distribuir de forma mais uniforme a tensão e o estresse sobre os pulmões, melhorar a relação ventilação/perfusão, melhorar a mecânica pulmonar de forma que 
ocorra a diminuição do tempo de VM e do índice de mortalidade. É importante que a pronação seja introduzida o mais rápido possível de preferência nas primeiras 24 horas, ou em até 48 horas, em pacientes com as trocas gasosas gravemente prejudicadas e em caso de SDRA. É indicado que o paciente permaneça nessa posição por ao menos 16 horas podendo chegar até 20 horas, antes de o posicionar novamente em posição supina (decúbito dorsal) (BORGES et al., 2020).

Um dos fatores primordiais para que execução do posicionamento ocorra de tranquila e segura, com uma probabilidade maior de sucesso é ter uma equipe treinada e experiente. Por isso, deve-se considerar que unidades que efetua a pronação, ofereça cursos e treinamentos para a toda equipe que realiza a manobra. (BORGES et al., 2020).

Alguns fatores são analisados para saber se a resposta a posição prona está sendo satisfatória ou não. Uma gasometria arterial deve ser feita 1hora após a pronação, para considerar se o paciente está respondendo ou não a técnica utilizada. O posicionamento deve ser conservado se o paciente apresentar uma boa resposta, ou seja, se ele apresentar um aumento de $20 \mathrm{mmHg}$ na $\mathrm{PaO} 2 / \mathrm{FiO} 2$ ou de $10 \mathrm{mmHg}$ na $\mathrm{PaO} 2$, lembrando que alguns pacientes podem responder ao processo de forma mais lenta podendo demorar até 6 horas para mostrar uma resposta favorável. Após 6 horas uma nova avaliação deve ser realizada, se o resultado for adequado mantém se a prono por 16 a 20 horas, caso contrário aconteça e o paciente não apresente uma boa resposta após a nova avaliação, volta o paciente a posição supina. A monitorização deve ser constante, sendo sugerido que uma nova avaliação ocorra a cada 6 horas (BORGES et al., 2020). 
Figura 1: Técnica é recomendada pela Organização Mundial da Saúde (OMS).

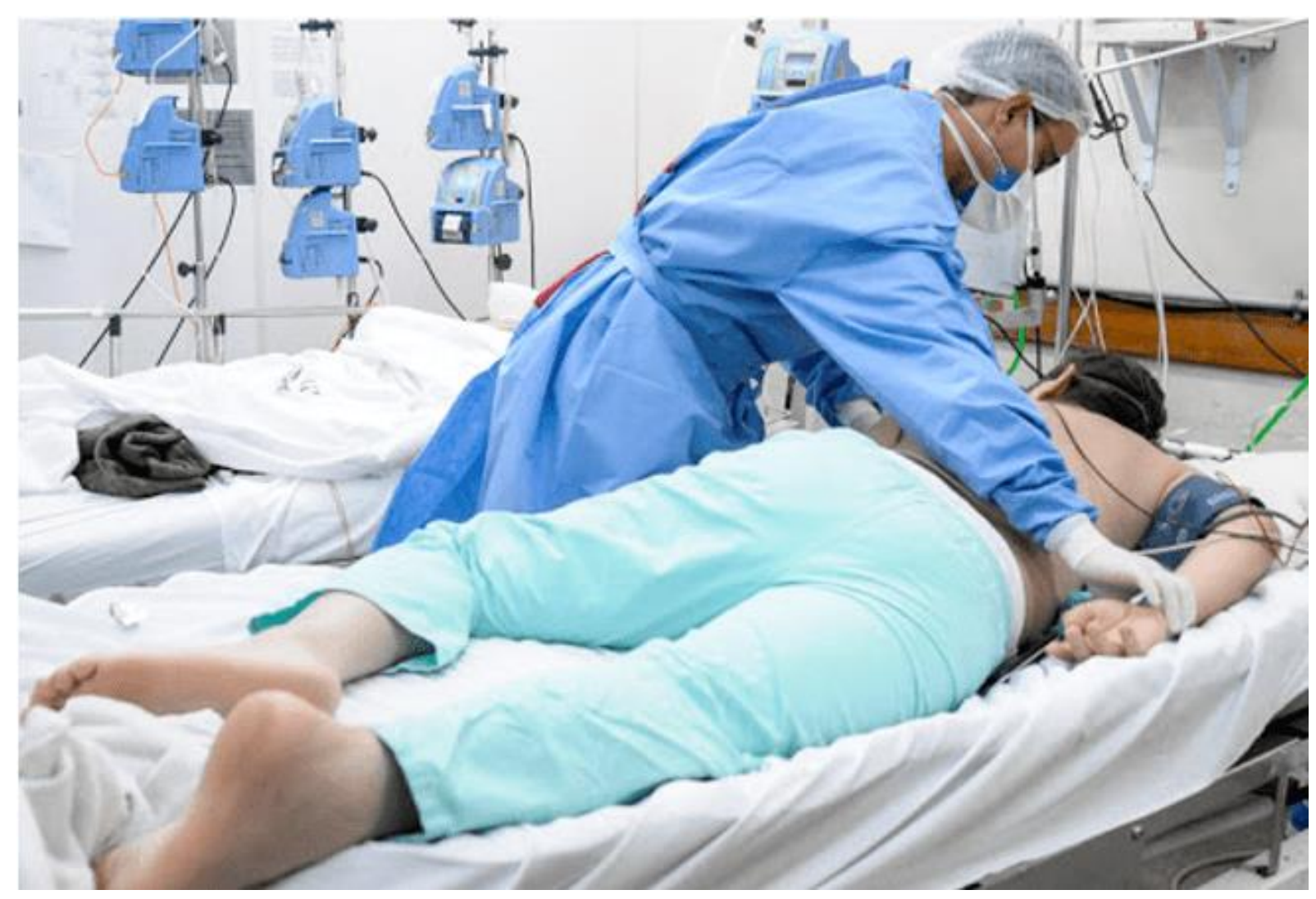

Foto: Geovana Albuquerque/Agência Saúde. 
Figura 2: Fluxograma de protocolo para posição prona.

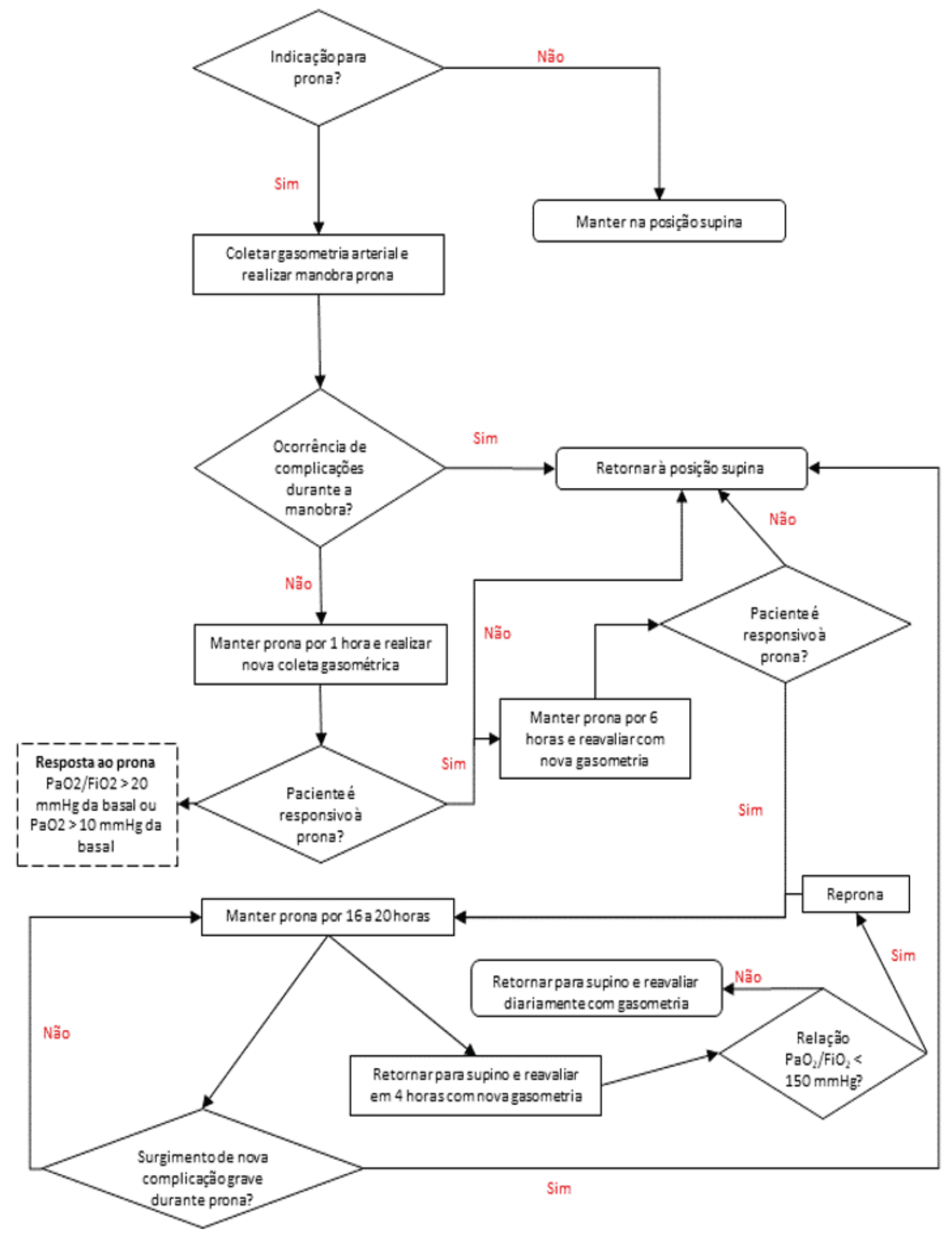

Fonte: Adaptado de BORGES e colaboradores. 


\section{CONSIDERAÇÕES FINAIS}

Visto que o COVID-19 trata-se de uma doença nova e as evidências sobre o assunto até o momento atual ainda são reduzidos, esta revisão bibliográfica abrange algumas das atribuições do fisioterapeuta em uma unidade de terapia intensiva, atuando na prevenção e no tratamento dos distúrbios respiratórios; na manutenção da força muscular (que pode ser prejudicada devido o período prolongado de repouso no leito) com a realização de exercícios passivos, ativo- assistido, ativos, mobilizações; utilizando a VNI como um dos recursos de tratamento do paciente com COVID-19 mantendo sempre uma monitorização criteriosa desse paciente; auxiliando na intubação; nos ajustes do ventilador mecânico - programando os parâmetros ventilatórios iniciais e buscando oferecer uma ventilação protetora com objetivo de diminuir o risco de uma lesão pulmonar - na monitorização da mecânica respiratória; no desmame da ventilação mecânica (VM) e durante a extubação; e os benefícios da posição prona ao paciente.

Vale destacar também a importância do fisioterapeuta ser capacitado, treinado e atualizado a respeito dos métodos utilizados como forma de tratamento.

\section{REFERÊNCIAS}

BORGES, D. L.; RAPELLO, G. V. G.; DEPONTI, G. N.; et al.. Prone position in the treatment of acute respiratory failure due to COVID-19. ASSOBRAFIR Ciência; Agosto 2020. DOI: https://doi.org/10.47066/2177-9333.AC20.covid19.011

BRANCO, A. B. A.C.; ARRUDA, K. D. S. A.; Atendimento psicológico de pacientes com COVID-19 em desmame ventilatório: proposta de protocolo. Revista Augustus; v. 25 n. 51; 2020. DOI: https://doi.org/10.15202/1981896.2020v25n51p335.

CASTRO, L. A.; RONCALLI, A.; CAMILLO, C. A. Desmame da ventilação mecânica na COVID-19. ASSOBRAFIR; Junho 2020. 
CORRÊA, T. D.; MATOS, G. F. J.; BRAVIM, B.A.; et al..Intensive support recommendations for critically-ill patients with suspected or confirmed COVID-19 infection. Einstein (São Paulo). 2020 May 29.

FRESCA et al. Recomendações relacionadas ao fluxo de atendimento para pacientes com suspeita ou infecção confirmada pelo COVID-19 em procedimentos cirúrgicos ou endoscópicos. SOBECC Março 2020.

FURLANETTO, K. C.; HERNANDES, N. A.; MESQUITA, R. B.. Recursos e técnicas fisioterapêuticas que devem ser utilizadas com cautela em pacientes com COVID-19. ASSOBRAFIR; Abril 2020.

GATTINONI, L.; CHIUMELLO, D.; CAIRONI, P.; et al. COVID-19 pneumonia: different respiratory treatments for different phenotypes. Intensive Care Med 46, 1099-1102 (2020). DOI: https://doi.org/10.1007/s00134-020-06033-2.

GÓMEZ, C. C.; RODRíGUEZ, Ó. P.; TORNÉ, M. L.; et al.. Clinical consensus recommendations regarding non-invasive respiratory support in the adult Patient with acute respiratory failure secondary to SARS-CoV-2 infection. Arch Bronconeumol. Julho de 2020; 56: 11-18.

GUIMARÃES, F. Approach of the physiotherapist in intensive care units in the context of the COVID-19 pandemic. Fisioter. Mov., Curitiba, v. 33, e0033001, 2020. DOI: http://dx.doi.org/10.1590/1980-5918.033.ED01.

GUO, T.; FAN Y.; CHEN M.; et al. Cardiovascular implications of fatal outcomes of patients with coronavirus disease 2019 (COVID-19). JAMA Cardio; March 27, 2020.

IMOTO et al. Cloroquina e hidroxicloroquina no tratamento da COVID-19: sumário de evidências. Com. Ciências Saúde 2020;31 Suppl 1:17-30.

LINDAHL, S. G. E.; Using the prone position could help to combat the development of fast hypoxia in some patients with COVID-19. Acta Paediatr. 2020 Aug;109(8):15391544. Epub 2020 Jun 17. DOI: https://dx.doi.org/10.1111\%2Fapa.15382 
MARTINEZ, B. P.; ANDRADE, F. M. D.; RONCALLI, A. et al..Intervenção na insuficiência respiratória aguda. ASSOBRAFIR; Março 2020.

MATOS, C. M. P.; SCHAPER, F. C.. Manejo fisioterapêutico para COVID-19 em ambiente hospitalar para casos agudos: recomendações para guiar a prática clínica. Dep. de Fisioterapia da Somiti. DOI: http://www.somiti.org.br/arquivos/site/comunicacao/noticias/2020/covid19/documentos/manejo-fisioterap-utico-para-covid-19.pdf.

MATTE, Darlan Laurício; ANDRADE, Flávio Maciel Dias de; MARTINS, Jocimar Avelar. et al.. O fisioterapeuta e sua relação com o novo SARS-CoV-2 e com a COVID19. ASSOBRAFIR CIÊNCIA; 2020 Ago;11(Supl 1):17-26. DOI: http://dx.doi.org/10.47066/2177-9333.AC20.covid19.002

MUSUMECI, M. M.; NOGUEIRA, I. C; ALCANFOR, T. A. F. et al.. Recursos fisioterapêuticos utilizados em terapia intensiva. ASSOBRAFIR; Maio 2020.

MUSUMECI, M. M.; MARTINEZ, B. P.; NOGUEIRA, I. C. et al.. Recursos fisioterapêuticos utilizados em unidades de terapia intensiva para avaliação e tratamento das disfunções respiratórias de pacientes com COVID-19. ASSOBRAFIR; Agosto 2020; 11 (Supl 1): 73-86.

PINTO, T. F.; CARVALHO, C .RF..SARS CoV-2 (COVID-19): lessons to be learned by Brazilian Physical Therapists. BJPT Associação Brasileira de Pesquisa e PósGraduação em Fisioterapia (ABRAPG-Ft). DOI: https://doi.org/10.1016/j.bjpt.2020.04.004.

REIS, M. A. S.. Suporte ventilatório COVID-19. AMIB - Associação de Medicina Intensiva Brasileira.

SEVERIN, R. et al.. Respiratory Muscle Performance Screening for Infectious Disease Management Following COVID-19: A Highly Pressurized Situation. O American Journal of Medicine. 2020. DOI: https://doi.org/10.1016/j.amjmed.2020.04.003. 
SILVA et al. Evidências científicas sobre fisioterapia e funcionalidade em pacientes com COVID-19 adulto e pediátrico. J Hum Growth Dev. 2020; 30(1):148-155. DOI:http://doi.org/10.7322/jhgd.v30.10086.

SOHRABI et al.. World Health Organization declares global emergency: A review of the 2019 novel coronavirus (COVID-19). Int J Surg. 2020 Apr; 76: 71-76.

WINCK, J. C.; AMBROSINO N. COVID-19 pandemic and non invasive respiratory management: Every Goliath needs a David. An evidence based evaluation of problems. Pulmonology. 2020 July-August; 26(4): 213-220.

WITTMER, V. L.; MOULIM, M.C.B.; GUIMARÃES, R. F.; et al.. Diário de exercícios fisioterapêuticos - pacientes estáveis com suspeita de COVID-19.Universidade Federal do Espírito Santo. 21 de abril de 2020.

THOMAS, P.; BALDWIN, C.; BISSETT, B.; et al.. Physiotherapy management for COVID-19 in the acute hospital setting: clinical practice recommendations. Journal of Physiotherapy. Volume 66, Issue 2, April 2020, Pages 73-82. DOI: https://doi.org/10.1016/j.jphys.2020.03.011

YEO, T. J.; WANG, Y. L.; LOW, T. T.. Have a heart during the COVID-19 crisis: Making the case for cardiac rehabilitation in the face of an ongoing pandemic. National Heart Centre, Singapore. Apr, 2020.

Enviado: Dezembro, 2020.

Aprovado: Janeiro, 2021. 